\title{
Dinámica temporal y espacial de los grupos funcionales del fitoplancton en un embalse tropical colombiano
}

\author{
Isabel Cristina Gil-Guarín* (iD, Silvia Lucía Villabona-González (D) y María Isabel Ríos- \\ Pulgarín (iD)
}

Grupo de Investigación en Limnología y Recursos Hídricos, Universidad Católica de Oriente, Rionegro, Colombia.

* Corresponding author: isabelcgg84@gmail.com

Received: 29/03/21 Accepted: 31/08/21

\section{RESUMEN}

Dinámica temporal y espacial de los grupos funcionales del fitoplancton en un embalse tropical colombiano

Los rasgos funcionales morfológicos, fisiológicos y ecológicos de las comunidades planctónicas en sistemas naturales han mostrado sensibilidad frente a la variabilidad hidrológica, no obstante, hay poca información respecto a la dinámica funcional del fitoplancton en embalses tropicales con alto tiempo de residencia. En este trabajo se estudió la composición y biomasa de los grupos funcionales del fitoplancton propuestos por Reynolds et al. (2002), sus cambios durante periodos hidrológicos contrastantes y a lo largo del gradiente espacial (longitudinal y vertical) y su relación con variables físicas y químicas en el embalse El Peñol-Guatapé. Se registraron siete grupos funcionales, de acuerdo con su preferencia en términos de luz, estado trófico y condiciones de mezcla: GF-F (Botryococcus braunni, Oocystis cf. lacustris), GF-Lm (Ceratium furcoides y Microcystis wesenbergii), GF-Lo (Chroococcus minutus, Peridinium gatunense y un organismo de Peridiniales), GF-P (Staurastrum rotula), GF-S1 (Oscillatoria limosa), GF-Y (Cryptomonas sp. y Parvodinium sp.) y GF-Sin (que incluyó a Cyclotella sp.). A nivel temporal, los grupos respondieron a factores asociados con la hidrodinámica, el clima y las sustancias suspendidas y disueltas. En la escala espacial-longitudinal las condiciones impuestas por el afluente principal, especialmente el gradiente de luz, fueron determinantes en la distribución de los grupos. En cuanto a la dimensión vertical, los procesos de mezcla favorecieron la permanencia de los organismos en la zona fótica, de acuerdo con sus rasgos morfológicos y fueron determinantes de su distribución.

Palabras clave: comunidad algal, rasgos funcionales, biomasa, gradiente longitudinal

\section{ABSTRACT}

Temporal and spatial dynamic of phytoplankton functional groups in a Colombian tropical reservoir

The morphological, physiological and ecological functional traits of planktonic communities have shown sensitivity to hydrological variability. However, there is little information regarding the functional dynamics of phytoplankton in tropical reservoirs with high residence time. In this study we analyse the composition and biomass of the functional groups of phytoplankton proposed by Reynolds et al. (2002), their changes during contrasting hydrological periods and along the spatial gradient (longitudinal and vertical), and its relationship with physical and chemical variables in the El Peñol-Guatapé reservoir. Seven functional groups were recorded, according to their preference in terms of light, trophic status and mixing conditions: GF-F (Botryococcus braunni, Oocystis cf. lacustris), GF-Lm (Ceratium furcoides and Microcystis wesenbergii), GF-Lo (Chroococcus minutus, Peridinium gatunense and Peridiniales organism), GF-P (Staurastrum rotula) GF-S1 (Oscillatoria limosa), GF-Y (Cryptomonas sp. and Parvodinium sp.) and GF-Sin (which included Cyclotella sp.). At temporal level, the groups responded to factors associated with hydrodynamics, climate, and suspended and dissolved substances. On the spatial-longitudinal scale, the conditions imposed by the main tributary, especially the light gradient, were decisive in the distribution of the groups. Regarding the vertical dimension, the mixing processes favoured the permanence of the organisms in the photic zone, according to their morphological features and were determinants for their distribution.

Key words: algal community, functional traits, biomass, longitudinal gradient 


\section{INTRODUCCIÓN}

En los últimos años en diferentes regiones del mundo se han construido un número importante de embalses con diferentes propósitos como suministro de agua potable, irrigación, navegación, control de inundaciones y generación de energía eléctrica (Zarfl et al., 2015, Grill et al., 2019). Esta tendencia es particularmente creciente en el neotrópico, debido a la riqueza hídrica y la topografía, generando importantes niveles de regulación sobre las cuencas (Carvajal-Quintero et al., 2017, Angarita et al., 2018). Contrario a un sistema natural, los embalses son sometidos a procedimientos de manejo operativo, que interfieren en sus características físicas, químicas y biológicas actuando en la escala temporal, longitudinal y vertical (Naselli-Flores, 2000, Rangel et al., 2012), con particular interferencia en el nivel de agua. Las oscilaciones en el nivel del agua y la pluviosidad representan un elemento significativo de la hidrodinámica común en casi todos los ecosistemas acuáticos. Tales fluctuaciones determinan la disponibilidad de luz y nutrientes y los cambios en la relación $\mathrm{Zm} / \mathrm{Zeu}$ (Zona de mezcla/zona eufótica), los cuales, en última instancia, influyen en la composición y estructura del fitoplancton (Bernhardt et al., 2008, Mac Donagh et al., 2009, Naselli-Flores, 2014).

Adicionalmente, la interacción entre el caudal de entrada y la operación del embalse provoca gradientes longitudinales en las condiciones físicas, químicas y biológicas que definen una configuración longitudinal del sistema. La presencia y éxito reproductivo del fitoplancton en los embalses están determinados por el efecto combinado de factores abióticos y bióticos como la temperatura, la turbidez, la concentración de nutrientes, el $\mathrm{pH}$, la herbivoría, los cambios en el nivel del agua, la estratificación térmica, la mezcla inducida por el viento y el caudal captado para generación en diferentes escalas espaciales y temporales (Reynolds, 2006). Por lo tanto, esta comunidad representa una efectiva herramienta de seguimiento para determinar la calidad del agua y ayudar a comprender las características y variaciones de estos ecosistemas acuáticos (Crossetti \& Bicudo, 2008, Costa et al., 2009;).

El fitoplancton se caracteriza por desarrollar rasgos funcionales morfológicos, fisiológicos y de comportamiento para sobrevivir en una amplia variedad de ambientes (Reynolds, 1988). Como respuesta a la interacción de diferentes factores, ciertas especies o grupos de especies podrían presentar un mejor desempeño ecológico frente a una serie particular de condiciones ambientales (Modesto et al., 2002) y dominar parcial o totalmente en un sistema (Roldán \& Ramírez, 2008), una vez hayan pasado a través de una serie de filtros ambientales que podrían limitar su desempeño (Naselli-Flores et al., 2016). Existe un creciente interés en evaluar las respuestas de las comunidades biológicas a la variabilidad hidrológica en sistemas regulados para la generación de energía, empleando los modelos de clasificación funcional del fitoplancton (Bortolini et al., 2019, Cupertino et al., 2019, Azevedo et al., 2020, Magalhães et al., 2020). Esto debido al potencial para explicar la organización de la comunidad en un sistema basándose en aspectos fisiológicos y morfológicos de los organismos, necesarios para su mejor desempeño ecológico. La agrupación de las especies de fitoplancton en grupos funcionales facilita la comprensión de su dinámica, a partir de la interacción de las variables ambientales con la presencia y el comportamiento de ciertos grupos, resultando en una herramienta útil cuando se intentan explicar y predecir las características y cambios en los embalses. El modelo de los grupos funcionales del fitoplancton propuesto por Reynolds et al. (2002) y revisado por Padisák et al. (2009) es una herramienta que ha facilitado la comprensión de estas características a diferentes escalas (Borges et al., 2008, Crossetti \& Bicudo, 2008, Becker et al., 2009, Padisák et al., 2009, Moreti et al., 2013, Bortolini et al., 2016, Souza et al., 2016). La razón de su éxito se debe a que permite simplificar largas listas de especies mediante la agrupación de taxones con características ecológicas similares en 31 asociaciones funcionales muy bien definidas, en función de las características de su hábitat, tamaño (biomasa), tolerancias y sensibilidades. En particular, la biomasa es una variable respuesta de gran importancia ecológica, debido a su papel en la oferta de alimento para los consumidores y la productividad del sistema (Straskraba \& Tundisi, 1999). La mayoría de los estudios sobre los gru- 
pos funcionales del fitoplancton se han llevado a cabo en sistemas templados, y su uso en sistemas tropicales es notablemente menos común, según lo describen Xiao et al. (2011); Hu et al. (2013) y Magalhães et al. (2021). En especial en embalses dendríticos con alto tiempo de residencia, en donde la variabilidad ambiental en el eje longitudinal no cumple necesariamente con el modelo general. En Colombia los únicos estudios con este enfoque incluyen el de López-Muñoz (2015) y Ospina-Calle \& Ramírez (2017). Ambos emplearon un intervalo de muestreo aproximado de dos meses, por lo que a la fecha no se ha publicado un estudio que haya considerado el corto periodo de tiempo en el que se reproducen las algas, proporcionando un modelo eficaz de conocimiento de procesos importantes en las comunidades planctónicas y en los ecosistemas en general (Moura et al., 2007).

Este estudio analizó la composición y biomasa de los grupos funcionales del fitoplancton propuestos por Reynolds et al. (2002) en el embalse Peñol-Guatapé (un embalse dendrítico con alto tiempo de residencia), con el objetivo de identificar el patrón de variación de dichos grupos durante periodos hidrológicos contrastantes y a lo largo del gradiente espacial (longitudinal y ver- tical) del embalse, así como establecer los principales factores determinantes de esta variación. Se hipotetiza que la expresión de biomasa de los grupos funcionales tiene diferencias sustanciales entre los periodos hidrológicos, entre los sectores del embalse (zonas de cola, transición y presa) y verticalmente en la zona fótica, en respuesta a variaciones estacionales en el nivel de agua, la luz y la temperatura.

\section{MATERIALES Y MÉTODOS}

\section{Área de estudio}

El embalse El Peñol-Guatapé se sitúa en el oriente del departamento de Antioquia en Colombia $\left(6^{\circ} 13^{\prime} 20^{\prime \prime} \mathrm{N}-75^{\circ} 10^{\prime} 11^{\prime \prime} \mathrm{O}\right)$ en jurisdicción de los Municipios de El Peñol, Guatapé y San Rafael, a $1887 \mathrm{msnm}$. Tiene un área de $6253.65 \mathrm{ha}$, una capacidad máxima hasta la cota de vertedero de 1240 millones $\mathrm{m}^{3}$, una profundidad máxima de $52 \mathrm{~m}$ y un tiempo de residencia aproximado de 140 días (Bedoya, 2017). Entre agosto y diciembre de 2014 se realizaron muestreos quincenales en cinco sitios de muestreo distribuidos en la zona de cola (SS1), en la zona de transición (SS2 y SS3) y en la zona de presa (SS4 y SS5) (Fig.
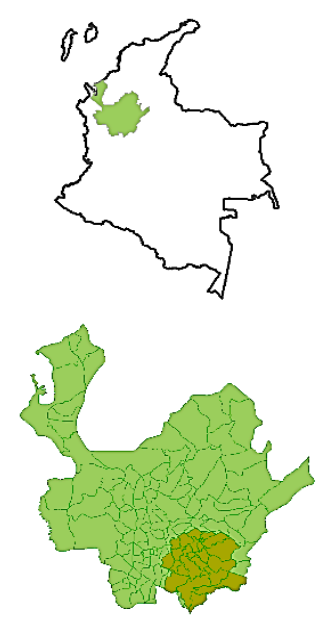
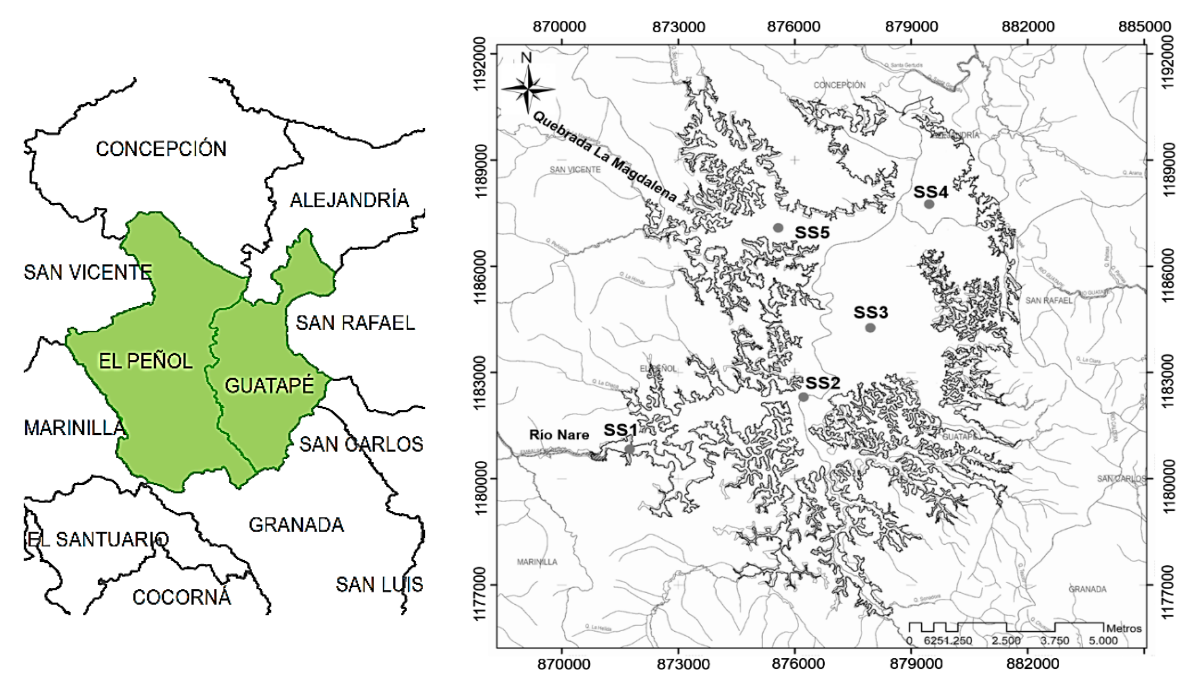

Figura 1. Localización del embalse El Peñol-Guatapé y de los sitios de muestreo. Location of El Peñol-Guatapé reservoir and sampling sites. 
1). El muestreo fue realizado en cuatro periodos: seco (agosto), transición a lluvias (septiembre), lluvias (octubre y noviembre) y transición a seco (diciembre).

\section{Datos ambientales}

Se utilizó la profundidad del disco de Secchi como indicador de transparencia y se determinaron in situ la temperatura del agua, la conductividad eléctrica, el oxígeno disuelto, la turbiedad $\mathrm{y}$ el $\mathrm{pH}$ mediante un perfilador CTD marca RBR modelo Maestro 3-13. En dos profundidades de la zona fótica, definida como 2.75 veces la profundidad del disco Secchi (Cole, 1994): a $100 \%$ de luz incidente (Subsuperficie) y a $1 \%$ de luz incidente $\left(E_{0}\right)$, se extrajeron muestras de agua con una botella Schindler para la medición del nitrógeno total (mg N/L), nitrógeno orgánico ( $\mathrm{mg} \mathrm{N}$ org/L), nitratos (mg $\left.\mathrm{N}_{-} \mathrm{NO}_{3}-\mathrm{L}\right)$, sulfatos $\left(\mathrm{mg} \mathrm{SO}_{4} 2 / \mathrm{L}\right)$, dióxido de carbono $\left(\mathrm{mg} \mathrm{CO}_{2} / \mathrm{L}\right)$, carbono orgánico total (mg COT/L) y turbiedad. Las concentraciones de nutrientes y sulfatos fueron estimadas en un cromatógrafo iónico (Metrohn 850 ic), el $\mathrm{CO}_{2}$ libre mediante el método titulométrico, el Carbono Orgánico Total (COT) por el método de combustión de alta temperatura y la turbiedad por el método nefelométrico (Baird \& Bridgewater, 2017). La información de las variables climatológicas e hidrológicas del río Nare fue proporcionada por Empresas Públicas de Medellín.

\section{Grupos funcionales del fitoplancton}

Las muestras de fitoplancton fueron recolectadas en la subsuperficie y al $1 \% \mathrm{E}_{\mathrm{o}}$ de atenuación de la irradiancia empleando una botella Schindler de cinco litros y estas fueron fijadas con una solución de lugol-ácido acético en una proporción de $2 \mathrm{ml}$ por cada $100 \mathrm{ml}$ de muestra (Ramírez, 2000). Se realizaron arrastres verticales para 1levar a cabo el análisis cualitativo del fitoplancton con una red de $25 \mu \mathrm{m}$ de tamaño de poro. Estas muestras fueron fijadas con solución transeau $(6$ partes de agua, 3 de alcohol al $70 \%$ y una de formol al $37 \%$ ). El análisis cuantitativo se realizó de acuerdo con Utermöhl (1958) y Lund et al. (1958) en un microscopio invertido Olympus CKX41, (el porcentaje de error implícito es me- nor al $7 \%$ (Lund et al., 1958). La densidad se registró en células/mL y se calculó utilizando la fórmula de Ross (1979). El volumen celular se determinó mediante el método del volumen celular medio $\left(\mu \mathrm{m}^{3}\right)$ siguiendo las recomendaciones de Hillebrand et al. (1999). En total, en cada una de las muestras se midieron 20 organismos de cada taxón seleccionados aleatoriamente. El biovolumen $\left(\mu \mathrm{m}^{3} / \mathrm{ml}\right)$ se obtuvo a partir del volumen celular medio, la media aritmética del número de células por individuo y la densidad obtenida mediante Ross (1979). Teniendo en cuenta el biovolumen por taxón y asumiendo que los organismos poseen una densidad igual a la del agua, las unidades de biomasa se reportan en mg/L (Edler, 1979, Wetzel \& Likens, 1991).

\section{Análisis de los datos}

Los taxones jerarquizados como dominantes, es decir aquellos que presentaron valores de dominancia y frecuencia mayores al promedio, de acuerdo con el modelo de Olmstead y Tukey (Sokal \& Rholf, 1981), fueron clasificados en los grupos funcionales empleando los criterios de Reynolds et al. (2002) y Padisák et al. (2009). Para verificar diferencias de la media o la mediana de la biomasa de los grupos funcionales entre los factores: periodos hidrológicos, sitio y profundidad de muestreo, se realizaron análisis de varianza (ANOVA) bifactoriales o Kruskal y Wallis cuando no se cumplieron los supuestos de la estadística paramétrica. Cuando se encontraron diferencias significativas se aplicaron las pruebas post hoc de Tukey o de U de Mann Whitney respectivamente. Para establecer los patrones generales de distribución de los grupos funcionales se realizó un Análisis Discriminante Canónico Generalizado con la biomasa de los grupos funcionales y las variables físicas y químicas. Se calcularon las puntuaciones y los vectores canónicos para la representación gráfica de las funciones discriminantes canónicas más importantes en términos de varianza explicada, permitiendo así la interpretación más simple en el espacio canónico de diferenciación entre la biomasa algal y la matriz ambiental. Los análisis fueron realizados con el programa R Wizard 2.3 (Guisande et al., 2014). 
Tabla 1. Valores de las variables físicas y químicas en la zona fótica: promedio ( $\bar{X})$, máximo (Max), mínimo (Min) y Coeficiente de Variación (CV, \%). Sub: subsuperficie; $1 \%$ : 1 \% de luz incidente; S: seco; LL: 1luvias; T: transición; NE: Número efectivo. Values of the physical and chemical variables in the photic zone: average $(\bar{X})$, maximum (Max), minimum (Min) and Coefficient of Variation (CV, \%). Sub: surface; $1 \%$ : 1 \% incident light; D: Dry; R: Rainy; T: Transition; EN: Effective Numbers.

\begin{tabular}{|c|c|c|c|c|c|}
\hline Variable & Promedio & Mínimo/muestra & Máximo/muestra & $\mathrm{DE}$ & CV (\%) \\
\hline Nitrógeno total (mg N/L) & 0.43 & 0.02/Sub-SS5-LL & $1.18 / 1 \%-S S 2-T$ & 0.25 & 57.25 \\
\hline Nitrógeno orgánico (mg N/L) & 0.25 & 0.01/1\%-SS5-LL & $0.90 /$ Sub-SS2-S & 0.17 & 70.45 \\
\hline Nitratos $\left(\mathrm{mg} \mathrm{NO}_{3} / \mathrm{L}\right)$ & 0.59 & $0.02 / 1 \%-S S 5-T$ & $2.63 / 1 \%-S S 2-L L$ & 0.73 & 123.62 \\
\hline Sulfatos $\left(\mathrm{mgSO}_{4} / \mathrm{L}\right)$ & 1.92 & $1.29 / 1 \%-S S 5-T$ & $3.64 / 1 \%-S S 3-T$ & 0.50 & 25.81 \\
\hline Dióxido de Carbono $\left(\mathrm{mgCO}_{2} / \mathrm{L}\right)$ & 3.67 & 0.00/Sub-SS1-T & $9.70 / 1 \%-S S 2-T$ & 1.94 & 52.85 \\
\hline Carbono Orgánico Total (mg C/L) & 3.09 & 1.00/Sub-SS2, SS4, SS5-S & 7.06/Sub-SS2-LL & 0.93 & 30.03 \\
\hline Turbiedad (NTU) & 2.90 & 0.71/Sub-SS5-T & 23.85/Sub-SS1-T & 3.81 & 131.64 \\
\hline Temperatura $\left({ }^{\circ} \mathrm{C}\right)$ & 23.28 & 21.38/1\%-SS5-LL & 25.31/Sub-SS1-LL & 0.98 & 4.20 \\
\hline Oxígeno disuelto $\left(\mathrm{mg} \mathrm{O}_{2} / \mathrm{L}\right)$ & 4.59 & 0.67/1\%-SS3-T & 8.28/Sub-SS1-S & 1.90 & 41.47 \\
\hline $\mathrm{pH}$ (unidades de $\mathrm{pH}$ ) & 7.31 & 6.49/1\%-SS4-T & 9.79/Sub-SS1-LL & 0.75 & 10.32 \\
\hline Conductividad $(\mu \mathrm{S} / \mathrm{cm})$ & 49.09 & 35.93/Sub-SS5-S & 75.35/1\%-SS3-T & 8.04 & 16.37 \\
\hline Transparencia (m) & 4.44 & 1.13/Sub-SS1-LL & 7.84/Sub-SS5-LL & 1.55 & 34.93 \\
\hline
\end{tabular}

\section{RESULTADOS}

\section{Variables físicas y químicas}

La precipitación varió entre $3.0 \mathrm{~mm} /$ día en el periodo seco (diciembre) y $8.6 \mathrm{~mm} /$ día durante el periodo de lluvias (noviembre) con un promedio de $5.3 \mathrm{~mm} /$ día. El nivel del agua mínimo fue $1881.9 \mathrm{~m}$ (a $8.1 \mathrm{~m}$ de la cota de vertimiento) y se presentó a finales del periodo de transición (septiembre), asociado a condiciones secas y un aumento en el caudal extraído para generación. Mientras que el nivel máximo fue $1885.1 \mathrm{~m}$ (a $4.9 \mathrm{~m}$ de la cota de vertimiento) y se presentó en el periodo de lluvias (noviembre), debido al aumento del caudal de entrada del río Nare. El embalse mantuvo este mismo nivel hasta el final del estudio, enmarcado en un periodo de transición a la época seca y disminución del caudal captado para generación. La temperatura del agua presentó un rango entre $21.38^{\circ} \mathrm{C}$ en SS5 (zona media) a finales de noviembre y $25.31{ }^{\circ} \mathrm{C}$ en SS1 (zona de cola) los primeros días de octubre, con un promedio de $23.28^{\circ} \mathrm{C}$. Durante el periodo de estudio la turbiedad fue mayor en la zona de cola $(\overline{\mathrm{X}}=10.72 \mathrm{NTU})$, en la zona de transición y de presa tuvo un promedio de 1.51 y 1.20 NTU, respectivamente. La disponibilidad de luz (Zeu:Zm) fue alta $(\geq 1)$ en todos los sitios y periodos de muestreo. Las concentraciones de oxígeno disuel- to oscilaron entre 0.67 y $8.28 \mathrm{mg} / \mathrm{L}$, las de $\mathrm{CO}_{2}$ entre 0.00 y $9.70 \mathrm{mg} / \mathrm{L}$, el rango para los sulfatos fue de 1.29 a $3.64 \mathrm{mg} / \mathrm{L}$ y el del carbono orgánico total de 1.00 a $7.06 \mathrm{mg} / \mathrm{L}$. En tanto, los nitratos, el nitrógeno total y el nitrógeno orgánico oscilaron entre 0.02 y $2.63 \mathrm{mg} / \mathrm{L} \mathrm{N}_{-} \mathrm{NO}_{3}, 0.02$ y $1.18 \mathrm{mg}$ $\mathrm{N} / \mathrm{L}$ y entre 0.01 y $0.90 \mathrm{mg} \mathrm{N}$ org/L respectivamente. El pH presentó valores entre 6.49 y 9.79 unidades y la conductividad eléctrica entre 35.93 y $75.35 \mu \mathrm{S} / \mathrm{cm}$ (Tabla 1).

\section{Dinámica de los grupos funcionales}

Los 12 taxones jerarquizados como dominantes de acuerdo con el modelo de Olmstead y Tukey representaron el $96.01 \%$ de la biomasa (905.64 mg/L). Se conformaron siete grupos funcionales, el grupo F (tolerante a condiciones de mezcla y alta transparencia, típico de sistemas mesotróficos) incluyó a Botryococcus braunni y Oocystis cf. lacustris, el Lm (característico de sistemas meso a eutróficos, oportunistas, reconocido por ser formador de floraciones y excelentes competidores, con baja presión por herbivoría) fue conformado por Ceratium furcoides y Microcystis wesenbergii. El grupo Lo (típico de sistemas mesotróficos, turbios no mezclados) estuvo conformado por Chroococcus minutus, Peridinium gatunense y un organismo indeterminado perteneciente al Orden Peridiniales. El grupo P (propio de sistemas 

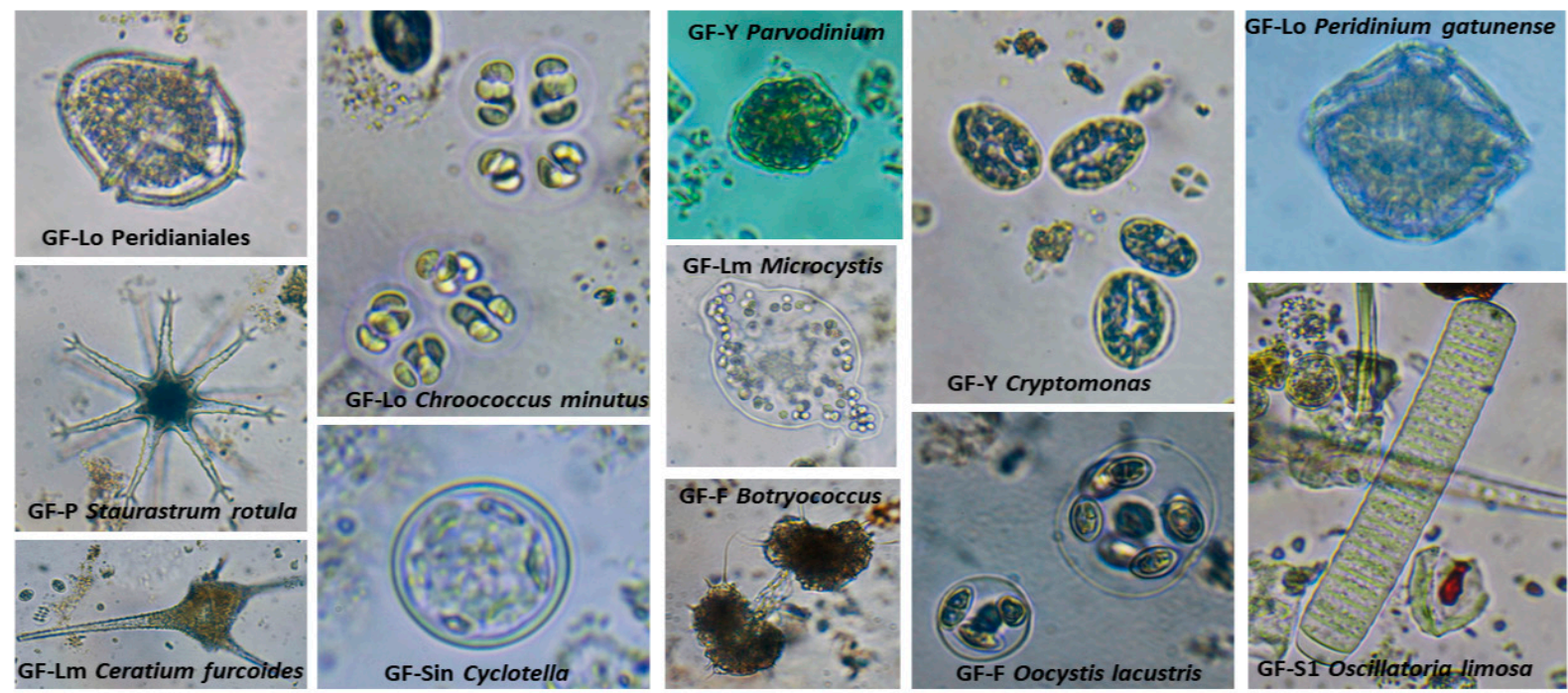

Figura 2. Registro fotográfico de los grupos funcionales del fitoplancton. Photographic record of phytoplankton functional groups.

Tabla 2. Análisis de varianza para la biomasa (mg/L) de los grupos funcionales del fitoplancton*. Grados de libertad (gl). Variance analysis for the biomass $(\mathrm{mg} / \mathrm{L})$ of phytoplankton functional groups *. Degrees of freedom (df).

\begin{tabular}{|c|c|c|c|c|c|c|c|c|c|c|c|}
\hline \multirow{2}{*}{ Grupo } & \multicolumn{4}{|c|}{ Campaña } & \multicolumn{3}{|c|}{ Estación } & \multicolumn{4}{|c|}{ Profundidad } \\
\hline & $\mathbf{F}$ & $X^{2}$ & gl & $p$ & $X^{2}$ & gl & $p$ & $\mathbf{F}$ & $X^{2}$ & gl & $p$ \\
\hline "Sin" & 5.13 & & 88 & $1.81 * 10^{5}$ & 1.763 & 3 & 0.62 & 0.00 & & 1 & 0.96 \\
\hline $\mathrm{F}$ & & 21.48 & 9 & 0.01 & 16.49 & 4 & 0.00 & & 4.59 & 1 & 0.03 \\
\hline $\mathrm{Lm}$ & & 19.10 & 9 & 0.02 & 27.64 & 4 & $1.476^{*} 10^{5}$ & & 0.02 & 1 & 0.88 \\
\hline Lo & & 6.57 & 9 & 0.68 & 15.37 & 4 & 0.00 & 11.66 & & 1 & 0.00 \\
\hline$P$ & & 17.22 & 9 & 0.05 & 8.35 & 4 & 0.08 & & 4.14 & 1 & 0.04 \\
\hline S1 & & 10.50 & 9 & 0.31 & 5.45 & 3 & 0.14 & & 14.45 & 1 & 0.00 \\
\hline $\mathrm{Y}$ & & 11.89 & 9 & 0.22 & 28.20 & 4 & $1.137^{*} 10^{5}$ & & 1.86 & 1 & 0.17 \\
\hline
\end{tabular}

meso a eutróficos, mezclados y turbios), lo integró Staurastrum rotula, el S1 Oscillatoria limosa (tolerante a ambientes mezclados y con menor transparencia), el grupo Y (propio de sistemas mesotróficos, con altas tasas de asimilación de nutrientes y crecimiento oportunista) fue conformado por Cryptomonas sp. y Parvodinium sp.), finalmente, el grupo denominado "Sin" (que incluyó una diatomea, identificada como Cyclotella sp., caracterizada por sus altas tasas de sedimentación y procesos de resuspensión en la columna de agua (Fig. 2). Ninguno de los grupos funcionales fue dominante en el fitoplancton del embalse. Los grupos Lm (26.87\%), Y (21.29 \%), F (20.22 \%) y Lo (19.99 \%), hicieron aportes muy similares a la biomasa total mientras los demás grupos no llegaron al $6 \%$.

La biomasa de los grupos funcionales GF-F, GF-Lm y GF-P varió en periodos hidrológicos, con un incremento en lluvias y transición a sequía. Por el contrario, el grupo GF-Sin, tuvo una biomasa significativamente mayor en el periodo seco. La biomasa de los grupos F y Lm fue significativamente diferente entre la zona de cola (máxima biomasa) y las zonas de transición y de presa. Mientras que el grupo Y tuvo significativamente menos biomasa en la cola del embalse. Los grupos F, Lo y P también mostraron diferencias 

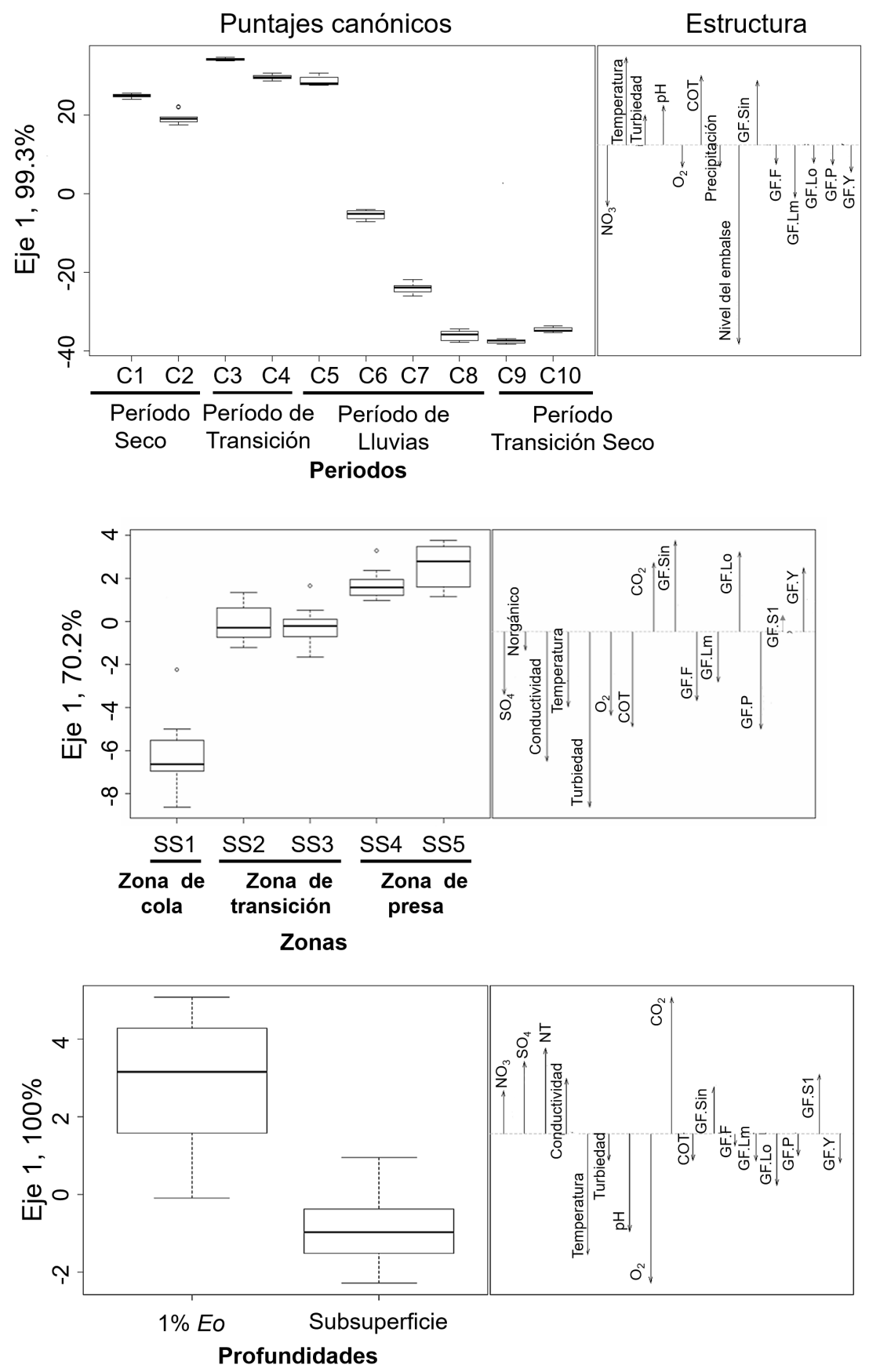

Figura 3. Primer eje canónico del Análisis Discriminante Canónico Generalizado para biomasa de los grupos funcionales del fitoplancton y las variables ambientales. $\mathrm{NO}_{3}$-Nitratos $\left(\mathrm{mg} \mathrm{NO}_{3} / \mathrm{L}\right)$. SO4-Sulfatos $\left(\mathrm{mg} \mathrm{SO}_{4} / \mathrm{L}\right)$. NT-Nitrógeno total (mg/L). NorgánicoNitrógeno orgánico $(\mathrm{mg} \mathrm{NO} / \mathrm{L})$. Conductividad eléctrica $(\mu \mathrm{S} / \mathrm{cm})$. Temperatura $\left({ }^{\circ} \mathrm{C}\right)$. Turbiedad $(\mathrm{NTU}) \cdot \mathrm{pH}($ unidades de $\mathrm{pH}) . \mathrm{O}_{2}-$ Oxígeno disuelto $(\mathrm{mg} / \mathrm{L})$. COT-Carbono orgánico total (mg COT/L). $\mathrm{CO}_{2}$-Dióxido de carbono (mg/L). Precipitación (mm/día). Nivel del embalse (m). Biomasa mg/L de los grupos funcionales (GF-Sin. GF-S1. GF-F. GF-Lm. GF-Lo. GF-P. GF-Y). First canonical axis of the Generalized Canonical Discriminant Analysis for biomass of phytoplankton functional groups and environmental variables. $\mathrm{NO}_{3}$-Nitrates $\left(\mathrm{mg} \mathrm{NO}_{3} / \mathrm{L}\right) . \mathrm{SO}_{4}$-Sulfates $\left(\mathrm{mg} \mathrm{SO}_{4} / \mathrm{L}\right)$. NT-Total Nitrogen $(\mathrm{mg} / \mathrm{L})$. Norganic-Organic Nitrogen $(\mathrm{mg} \mathrm{NO} / \mathrm{L})$. Electrical conductivity $(\mu \mathrm{S} / \mathrm{cm})$. Temperature $\left({ }^{\circ} \mathrm{C}\right)$. Turbidity (NTU). $\mathrm{pH}$ ( $\mathrm{pH}$ units). O $\mathrm{O}_{2}$-Dissolved oxygen (mg/L). TOC-Total organic carbon (mg TOC/L). $\mathrm{CO}_{2}$-Carbon dioxide $(\mathrm{mg} / \mathrm{L})$. Precipitation $(\mathrm{mm} /$ day). Reservoir level $(\mathrm{m})$. Biomass $\mathrm{mg} / \mathrm{L}$ of functional groups $(\mathrm{FG}-\mathrm{Sin}$. FG-S1. FG-F. FG-Lm. FG-Lo. FG-P. FG-Y). 
verticales importantes entre la subsuperficie y el $1 \%$ de Eo, donde su biomasa disminuyó. Contrario a los demás grupos, $\mathrm{S} 1$ presentó su mínima biomasa en la subsuperficie (Tabla 2).

Los análisis discriminantes mostraron que los periodos de muestreo, y especialmente las profundidades, presentaron mayor variabilidad ambiental que biológica y los grupos funcionales hicieron una contribución moderada a la varianza.

En la escala temporal, el Análisis Discriminante Canónico (ADC) clasificó correctamente el $85.39 \%$ de los casos por validación cruzada y explicó el $99.30 \%$ de la varianza de los datos acumulada en el primer eje canónico. Este análisis muestra que las campañas realizadas durante el periodo seco y de transición a las lluvias fueron discriminadas por mayor biomasa de organismos del grupo funcional Sin (GF-Sin), asociado a aguas más cálidas, con mayor $\mathrm{pH}$, carbono orgánico total (COT) y turbiedad. Las últimas campañas de monitoreo, correspondientes al periodo de lluvias y de transición al periodo seco se diferenciaron por el aumento del nivel del agua y los nitratos, así como de la biomasa del grupo funcional Lm. El análisis mostró alta estabilidad en la biomasa de los demás grupos funcionales en la escala temporal (Fig. 3).

En la escala espacial longitudinal (Fig. 3) el ADC arrojó un $67 \%$ de clasificación con validación cruzada y una varianza explicada del $70.20 \%$ para el primer eje canónico. El análisis mostró la discriminación de la zona de cola (SS1) por el incremento de la biomasa del grupo funcional $\mathrm{P}$ asociado a una mayor turbiedad, conductividad eléctrica, carbono orgánico total y concentración de oxígeno disuelto. El aumento de la biomasa de los grupos funcionales F y Lm tendió a diferenciar la zona de transición (SS2 y SS3) asociada a unas condiciones intermedias de turbiedad, y conductividad eléctrica, al aumento en la temperatura y concentración de sulfatos $\left(\mathrm{SO}_{4}\right)$. Los grupos funcionales "Sin", Lo y Y discriminaron la zona de presa (SS4 y SS5), con mayor concentración de $\mathrm{CO}_{2}$.

En la escala vertical, el porcentaje de clasificación con validación cruzada del EDC fue $90.77 \%$ y el primer eje canónico explicó el $100 \%$ de la varianza. Los grupos que más contribuyeron a esta discriminación fueron Lo, S1 y "Sin"; el gru- po Lo diferenció levemente la subsuperficie y los grupos S1 y "Sin" la profundidad al $1 \% \mathrm{E}_{\mathrm{o}}$. La subsuperficie también fue discriminada por mayores valores de oxígeno disuelto, temperatura y $\mathrm{pH}$, mientras el $1 \% \mathrm{E}_{\mathrm{o}}$ presentó las máximas concentraciones de dióxido de carbono $\left(\mathrm{CO}_{2}\right)$, nitrógeno total $(\mathrm{NT})$, nitratos $\left(\mathrm{NO}_{3}\right)$ y sulfatos $\left(\mathrm{SO}_{4}\right)$, así como la mayor conductividad eléctrica (Fig. 3).

\section{DISCUSSION}

Los resultados encontrados durante este estudio mostraron variaciones espaciales y temporales en las condiciones ambientales en este ecosistema, así como la respuesta de los atributos de la comunidad fitoplanctónica a esta heterogeneidad. De acuerdo con Naselli-Flores \& Barone (2000) y Wang et al. (2011) los cambios en la pluviosidad y el régimen hidráulico son los principales determinantes de la acumulación de biomasa del fitoplancton y su variación temporal en los embalses, pues alteran variables fundamentales para su proliferación, funcionamiento y adaptación como son el nivel del agua, la incidencia de luz y su relación con los eventos de mezcla, las sustancias suspendidas, la biodisponibilidad de iones y sustancias disueltas, el metabolismo y balance entre los gases disueltos y la acidez y alcalinidad del agua.

La influencia de algunas de estas condiciones sobre los rasgos funcionales de las especies discriminó dos períodos contrastantes, el primero durante la temporada de menor precipitación (campañas 1 a 5) que generó un menor ingreso de caudal del afluente principal (río Nare) y por tanto también menor nivel del agua y el segundo, durante las últimas campañas (C6 a C10), cuando incrementaron la pluviosidad, el caudal de ingreso del afluente y el nivel. En el primero, la mayor expresión de biomasa del grupo "Sin" conformado por el género Cyclotella sp. (género más representativo del grupo) coincidió con aguas más cálidas y turbias y con mayor $\mathrm{pH}$ y contenido de carbono orgánico. Este grupo se caracteriza por sus altas tasas de sedimentación ocasionadas por el peso de su frústula de sílice. Como consecuencia, es incapaz de regular su posición en la columna de agua y requiere de procesos de resuspensión para evitar pérdidas por hundimiento. Estos 
procesos de resuspensión podrían haber sido facilitados por el ingreso de la pluma del afluente principal (río Nare), la cual, de acuerdo con la descripción de Bedoya (2017), se desplazó como una corriente intrusiva que se extendió hasta la zona de presa a una profundidad equivalente a la de la zona fótica (17 metros aproximadamente) durante dicho periodo. Adicionalmente, también se presentaron movimientos de agua asociados al aumento del caudal extraído para generación que también pudieron favorecer la resuspensión. En la escala espacial, la presencia de la captación de agua para generación eléctrica en la zona de presa propició la mezcla del agua y procesos de resuspensión que hicieron que diatomeas sedimentables como Cyclotella sp. se adaptaran mejor a estas características, tal como fue evidenciado por el análisis discriminante. En el segundo periodo, el incremento de las lluvias favoreció la disponibilidad de iones para el crecimiento de especies oportunistas capaces de aprovechar esta condición gracias a sus altas tasas de crecimiento (Litchman et al., 2015). Como resultado, dichas especies aumentaron su biomasa, especialmente los grupos Lm y Y, durante la campaña 6, posterior al aumento de las precipitaciones y de la concentración de la forma de nitrógeno más asimilable por las algas $\left(\mathrm{NO}_{3}\right)$. Estos factores favorecieron la consolidación de la biomasa del grupo funcional $\mathrm{Lm}$ en las últimas campañas $(\mathrm{C} 8, \mathrm{C} 9$ y C10) después de varias semanas de lluvias. En otros embalses tropicales como Riogrande II, se ha observado el aumento de la biomasa de $\mathrm{Mi}$ crocystis sp., una de las especies que conforman del grupo Lm, como consecuencia de la llegada de iones a la columna de agua, posterior a un periodo de lluvias fuertes que favorecieron el lavado de la cuenca y la resuspensión del sedimento (Palacio-Betancur, 2014). Varios autores reportan que el grupo Lm es usualmente encontrado en sistemas meso a eutróficos (Reynolds, et al., 2002, Naselli-Flores \& Barone, 2003, 2005; Hoyer et al., 2009). Debido a que toleran un amplio rango en las condiciones ambientales, pueden regular su posición en la columna de agua, tienen baja presión por herbivoría y una alta dispersión, las especies de este grupo son consideradas excelentes competidores (Salmaso, 2003, Codd et al., 2005, Aubriot et al., 2009, Bustamante-Gil et al., 2012,
Boltovskoy et al., 2013). El grupo Y conformado por Cryptomonas sp. y Parvodinium sp. es muy común en sistemas donde la presión por herbivoría es baja (Padisák et al., 2009), ya que son nutricionalmente pobres y en ocasiones tóxicas. De acuerdo con Reynolds et al. (2002), es tolerante a ambientes donde los coeficientes de atenuación de luz son altos. En este estudio, predominó en un entorno con alta disponibilidad de luz como fue expresado por la relación $\mathrm{Zeu} / \mathrm{Zm}$ mayor a uno en todos los casos. Estudios como el de Jones (2000) y Wang et al., (2011) resaltan su capacidad para adquirir nutrientes por mixotrofia y así, desarrollarse en condiciones de luz limitada. Además, la presencia de flagelos les permite mejorar la competitividad al permitir la migración vertical en la columna de agua (Bovo-Scomparin \& Train, 2008). Su estrategia de supervivencia se encuentra entre colonizador C-estratega y tolerante R-estratega (Dos Santos \& Calijuri, 1998), lo cual le permite permanecer en la columna de agua por periodos de tiempo largos y aumentar su biomasa luego de una perturbación, dada su condición de oportunista y sus altas tasa de crecimiento (Crossetti \& Bicudo, 2005, Salmaso \& Tolotti, 2010). Como ocurrió en este estudio, su presencia en todos los periodos, pero especialmente su incremento después del periodo de lluvias, denota su nivel de ubicuidad y ventajas competitivas respecto a otros grupos (Bovo-Scomparin \& Train, 2008, Borges et al., 2008, Wang et al., 2011).

El análisis discriminante mostró que ciertos grupos funcionales fueron característicos de las zonas del embalse lo que demuestra que los factores ambientales propios de cada una actuaron sobre los rasgos de las especies para estructurar la comunidad fitoplanctónica (Zhang et al., 2015). Este marcado gradiente longitudinal de la biomasa de los grupos se relacionó con procesos de mezcla y turbulencia, estado trófico y disponibilidad de luz, que dividió al embalse en tres zonas. La primera es la zona de cola (SS1), dominada por el grupo funcional $\mathrm{P}$, que es reportado comúnmente en ambientes eutróficos donde, la turbulencia, las sustancias suspendidas y disueltas como los nutrientes y el carbono orgánico también son altas (Aguirre-Ramírez et al., 2007, Reynolds et al., 2002, Pádisak et al., 2009). Esta descripción responde estrechamente a las condiciones de esta 
zona del embalse Peñol-Guatapé, caracterizada por una mayor velocidad del agua, menor tiempo de residencia, altas concentraciones de nutrientes biodisponibles y una mayor turbidez, dada su ubicación en el área de ingreso del principal afluente del sistema (Thornton et al., 1990). En segundo lugar, la zona de transición, donde el flujo avanza en dirección a la presa y normalmente aumenta la biomasa del fitoplancton, disminuye la velocidad del flujo y la turbidez y aumenta el tiempo de residencia (Thornton et al., 1990). Debido a que tanto la luz como los nutrientes están más disponibles en esta zona, se considera la región más productiva y fértil de un embalse. Allí se observó el incremento de la biomasa de los grupos $\mathrm{F} y$ Lm. El grupo F fue propuesto como característico de ambientes mesotróficos con alta disponibilidad de luz (Reynolds et al., 2002, Padisák, 2009) e influenciado por la mezcla resultante de la entrada del afluente, del caudal extraído para generación y del viento, que ocasionan movimientos horizontales y verticales de la columna de agua. Becker et al. (2010) y Huichao-Dai et al. (2013) registraron la presencia de organismos del grupo $\mathrm{F}$ en condiciones muy similares a las descritas. Como fue mencionado, el grupo Lm fue propuesto para sistemas turbios con alta concentración de nutrientes; sin embargo, la ocurrencia de este grupo con preferencias ambientales contrastantes en esta zona del embalse se explica porque está conformado por organismos oportunistas, con tasas rápidas de asimilación de nutrientes y con alta capacidad de desplazamiento vertical en la columna de agua. Aunque su presencia ha sido relacionada con un grado de trofia alto, los representantes de este grupo pueden desarrollarse en un rango muy amplio de condiciones.

En tercer lugar, la zona de presa, la cual generalmente tiene la velocidad de flujo más baja, mayor tiempo de residencia, concentraciones más bajas de nutrientes y sedimentos suspendidos, mayor transparencia del agua y una zona fótica más profunda que la zona de transición (Thornton et al., 1990). Como se mencionó, el grupo "Sin" (Cyclotella sp.), así como Lo y Y, se adaptaron mejor a estas características. El grupo Lo incluye dinoflagelados grandes, generalmente encontrados en sistemas mesotróficos, turbios no mezclados (Reynolds et al., 2002). Sin embargo, en este caso, el aumento de la biomasa en esta zona demuestra un alto grado de adaptabilidad a los procesos de mezcla y no se ajusta estrictamente al modelo propuesto por Reynolds et al. (2002). La presencia de flagelos contribuye a la movilidad del organismo y a la renovación del agua a su alrededor, facilitando su contacto con los nutrientes más próximos a la célula (Marques-Lopes et al., 2005). En consecuencia, la motilidad pudo ser una ventaja que permitió la movilidad de los organismos desde áreas agotadas en nutrientes que limitan la producción primaria, como la zona de presa.

En la escala vertical esta capacidad de regular su posición en la columna de agua por locomoción activa y tolerar alta temperatura e iluminación (Borics et al., 2005) permite explicar la leve asociación del grupo Lo con la subsuperficie. Aunque según Grigorszky et al. (2003) los dinoflagelados evitan permanecer en esta zona de la columna de agua, durante este estudio no se observó este patrón en su comportamiento. Al igual que Lo, el grupo funcional Y, también se encuentra adaptado a una amplia gama de condiciones ambientales (Reynolds et al., 2002, Rodrigues et al., 2005, Train et al., 2005, Pivato et al., 2006). En los embalses catalanes Foix, Sau, La Baells y La Llosa del Cavall, Caputo et al. (2008) también encontraron que los grupos funcionales del fitoplancton respondieron a la disponibilidad de luz y nutrientes en un gradiente longitudinal. Un resultado similar fue reportado por Borges et al. (2008) en el embalse Capivari, y por Souza et al. (2016) en el embalse Santa Laura, en tanto, en el embalse Segredo este gradiente fue casi imperceptible debido al bajo tiempo de retención hidráulica Borges et al. (2008).

En embalses tropicales la temperatura del agua, la dinámica de los gases, sustancias disueltas como los nutrientes y la disponibilidad de luz, son los principales agentes de cambio en la composición y en las estrategias funcionales del fitoplancton en la escala vertical (Gomes-Barbosa et al., 2011, Lira et al., 2014). En contraste con estas investigaciones, y en concordancia con lo que hallaron Soares-Vieria et al. (2015) y López-Muñoz (2015), en esta investigación se observó que la distribución vertical de la mayoría de los grupos funcionales del fitoplancton no mostró una dependencia clara de las variables abióticas medi- 
das y no hubo ninguna preferencia por alguna de las profundidades. Posiblemente se debe a que los grupos registrados presentan algún tipo de adaptación para posicionarse dentro de la columna de agua y para controlar la flotación y evitar pérdidas por hundimiento. Adicionalmente, los movimientos del agua por la acción del viento pueden inducir a movimientos horizontales y verticales de la columna de agua que favorecen sustancialmente la permanencia de los organismos en el epilimnion, ya que ejerce un efecto homogeneizador (Matsumura-Tundisi et al., 2010).

Por otra parte, tal como lo menciona Reynolds (1993), es muy importante tener en cuenta la escala de tiempo, la cual es muy corta en el fitoplancton ya que estos factores actúan más rápido que el tiempo que se tarda esta comunidad para responder a estos estímulos. Richerson et al. (1970) indican que, si la velocidad en las variaciones del medio fueran lo suficientemente lentas en relación con la tasa reproductiva de las algas, se generarían nichos diferentes que derivarían en el aumento en la biomasa de los grupos que respondan mejor a estas condiciones.

Los grupos funcionales S1 y "Sin" se caracterizan por su alta tolerancia a ambientes mezclados y turbios (Padisák, 2009). El grupo S1 estuvo conformado por una cianobacteria con una alta relación superficie-volumen y peso, y el grupo "Sin" por un organismo con frústula de sílice. Ambos organismos, como consecuencia de estos rasgos morfológicos, experimentan altas tasas de sedimentación, lo cual explicaría una mayor biomasa de estos grupos en la profundidad al 1\%. Para contrarrestar estas pérdidas requieren de procesos de mezcla que los mantengan en suspensión dentro de la zona fótica donde reciben luz suficiente para el proceso de la fotosíntesis y conservación de su biomasa (Barbosa \& Padisak, 2002).

Por último, los resultados de este estudio coinciden con los encontrados por Magalhães et al. (2021), en embalses tropicales con alto tiempo de residencia localizados por debajo de $500 \mathrm{msnm}$. En los cuales la luz (turbiedad) y la concentración de nutrientes, $\mathrm{pH}$ y temperatura son las variables determinantes de la distribución espacial de los grupos funcionales del fitoplancton. Este patrón se mantiene, aun considerando diferencias importantes en el embalse Peñol-Guatapé como su mayor altitud, largo tiempo de operación desde su construcción y alto grado de desarrollo de la línea costera (Dendrítico), condiciones que promueven una alta heterogeneidad espacial. En ambos estudios los modelos empleados describieron eficientemente la variación espacial de los grupos funcionales de acuerdo con el modelo de Thorton et al. (1990) favorecidos por el alto tiempo de residencia de los sistemas. De manera que este parece ser un factor clave en la dinámica del fitoplancton en embalses tropicales, independientemente de su altitud o morfometría. Dilucidar la prevalencia de este patrón es un aporte significativo al conocimiento de los embalses tropicales.

\section{CONCLUSIONES}

Los resultados encontrados en este estudio permitieron verificar la sensibilidad de la expresión de la biomasa de algunos grupos funcionales del fitoplancton a los cambios ambientales de diferentes escalas en sistemas regulados tropicales. Los resultados soportan las hipótesis de una variación temporal y espacial longitudinal, al evidenciar que la distribución de la biomasa de algunos grupos funcionales respondió a las variables asociadas con la hidrodinámica del embalse y a las condiciones impuestas por el ingreso del afluente principal, especialmente el gradiente de luz y de sustancias suspendidas y disueltas. Confirmando que el tiempo de residencia resulta un factor clave en la dinámica del fitoplancton en embalses tropicales donde este tiempo es alto, independientemente de su altitud o morfometría.

En cuanto a la dimensión vertical, los resultados no soportan la hipótesis prevista, dado que los rasgos morfológicos de algunos de los grupos fueron determinantes de su distribución en la zona fótica. En general, los resultados muestran tendencias poco pronunciadas debido a que no existe limitación por luz, la mayoría de los grupos presentan estrategias para desplazarse a lo largo de la columna de agua y los procesos de mezcla favorecen la permanencia de los organismos en el epilimnion.

\section{AGRADECIMIENTOS}

Este estudio fue parte de un proyecto de investigación financiado por Empresas Públicas de 
Medellín (EPM) a través de una beca de maestría otorgada al primer autor y el apoyo a algunas de las actividades de investigación. Los autores agradecen a EPM por la información suministrada y al equipo del convenio Análisis ecosistémico de factores determinantes para la ordenación del uso pesquero y acuícola en el embalse Peñol - Guatapé por su apoyo durante las campañas de muestreo.

\section{REFERENCIAS}

Aguirre, N. J., Palacio, J. A., \& Ramírez J. J. (2007). Características limnológicas del embalse El Peñol-Guatapé. Revista de Ingenierías Universidad de Medellín, 6(10), 53-66. http://www.scielo.org.co/pdf/rium/v6n10/ v6n10a05.pdf

Angarita, H., Wickel, A., Sieber, J., Chavarro, J., Maldonado-Ocampo, J., Herrera, G., Delgado, J., \& Purkey, D. (2018). Basin-scale Impacts of Hydropower Development on the Mompós Depression Wetlands, Colombia. Hydrology and Earth System Sciences, 22, 2839-2865. DOI: 10.5194/hess-22-2839-2018

Aubriot, L., Bonilla, S., \& Kruk, C. (2009). Cianobacterias planctónicas: factores que regulan su crecimiento. En: S. Bonilla, (Ed.), Cianobacterias Planctónicas del Uruguay. Manual para la identificación y medidas de gestión. Parte I-Generalidades (5-11). Montevideo, Uruguay.

Azevedo, A. D. S., Moura, A. N., Aragão-Tavares, N. K. C., \& Dantas, E. W. (2020). Taxonomic and functional approaches to phytoplankton in ecosystems with different coverage of aquatic plants. Brazilian Journal of Botany, 43, 665675. DOI: 10.1007/s40415-020-00626-3

Baird, R., \& Bridgewater, L. (2017). Standard methods for the examination of water and wastewater. American Public Health Association. Washington, D.C. Estados Unidos.

Barbosa, F. A. R., \& Padisák, J. (2002). The forgotten lake stratification pattern: atelomixis, and its ecological importance. Verhandlungen des Internationalen Verein Limnologie, 28, 13851395. DOI: 10.1080/03680770.2001.11902683

Bedoya-Cardona, C. M. (2017). Dinámica espacial y temporal de la estructura térmica del embalse Peñol-Guatapé. (Tesis de maestría. Uni- versidad Nacional de Colombia, Colombia). https://repositorio.unal.edu.co/handle/unal/ 63848

Bernhardt, J., Elliott, J. A., \& Jones, I. D. (2008). Modelling the effects on phytoplankton communities of changing mixed depth and background extinction coefficient on three contrasting lakes in the English Lake District. Freshwater Biology, 53(12), 2573-2586. DOI: 10.1111/j.1365-2427.2008.02083.x

Becker, V., Cardoso, L. S. C., \& Huszar, V. L. M. (2009). Diel variation of phytoplankton functional groups in a subtropical reservoir in southern Brazil during an autumnal stratification period. Aquatic Ecology, 43 (2), 285-293. DOI: $10.1007 / \mathrm{s} 10452-008-9164-0$

Becker, V., Caputo, L., Ordoñez, J., Marcé, R., Armengol, J., Crossetii, L.O., \& Huszar, V. (2010). Driving factors of the phytoplankton functional groups in a deep Mediterranean reservoir. Water Research, 44(11), 3345-3354. DOI: $10.1016 /$ j.watres.2010.03.018

Borics, G., Grigorszky, I., Padisák, J., Barbosa, F., \& Doma, Z. (2005). Dinoflagellates from tropical Brazilian lakes with description of Peridinium brasiliense sp. nova. Algological Studies, 118, 47-61. DOI: 10.1127/1864-1318/ 2006/0118-0047

Boltovskoy, A., Echenique, R., \& Guerrero, J. M. (2013). Sucesivas invasiones de especies de Ceratium (Dinophyceae) en Sudamérica: Un proceso que lleva dos décadas. Boletín de la Sociedad Argentina de Botánica, 48 (Supl): 27. https://www.researchgate.net/publication/ 256463976

Borges, P. A. F., Train, S., \& Rodrigues, L. C. (2008). Spatial and temporal variation of phytoplankton in two subtropical Brazilian reservoirs. Hydrobiologia, 607(1), 63-74. DOI: 10.1007/s10750-008-9367-3

Bortolini, J. C., Train, S., \& Rodrigues, L. C. (2016). Extreme hydrological periods: effects on phytoplankton variability and persistence in a subtropical floodplain. Hydrobiologia, 763(1), 223-236. DOI: 10.1007/s10750-0152378-y

Bortolini, J., Da Silva, P. R., Baumgartner, G., \& Bueno, N. (2019). Response to environmental, spatial, and temporal mechanisms of the phy- 
toplankton metacommunity: comparing ecological approaches in subtropical reservoirs. Hydrobiologia, 830, 45-61. DOI: 10.1007/ s10750-018-3849-8

Bovo-Scomparin, V. M. \& Train, S. (2008). Longterm variability of the phytoplankton community in an isolated floodplain lake of the Ivinhema River State Park, Brazil. Hydrobiologia, 610(1), 331-344. DOI: 10.1007/ s10750-008-9448-3

Bustamante-Gil, C., Ramírez, J. J., Boltovskoy, A., \& Vallejo, A. (2012). Spatial and temporal change characterization of Ceratium furcoides (Dinophyta) in the equatorial reservoir Rio Grande II, Colombia. Acta limnologica Brasiliensia, 24(2), 207-219. DOI: 10.1590/ S2179-975X2012005000039

Caputo, L., Naselli-Flores, L., Ordoñez, L. J., \& Armengol, J. (2008). Phytoplankton distribution along trophic gradients within and among reservoirs in Catalonia (Spain). Freshwater Biology, 53, 2543-2556. DOI: 10.1111/j.13652427.2008.02082.x

Carvajal-Quintero, J. D., Januchowski-Hartley, S. R., Maldonado-Ocampo, J. A., Jezequel, C., Delgado, J., \& Tedesco, P. (2017). Damming Fragments Species Ranges and Heightens Extinction Risk. Conservation Letters, 10(6), 708-716. DOI: 10.1111/conl.12336

Codd, G. A., Lindsay, J., Young, F. M., Morrison, L. F., Metcalf, J. S. (2005). Harmful Cyanobacteria. From mass mortalities to management measures. En: J. Huisman, H.C. Matthijs \& P.M. Visser. (Eds.), Harmful Cyanobacteria. (pp. 1-24). Springer, Holanda

Cole, G. A. (1994). Textbook of Limnology. Waveland Press Inc., Prospect Heights, Illinois. Estados Unidos

Costa, L. S., Huszar, V. L. M., \& Ovalle, A. R. (2009). Phytoplankton functional groups in a tropical estuary: hydrological control and nutrient limitation. Estuaries and Coasts, 32(3), 508-521. DOI: 10.1007/s12237-009-9142-3

Crossetti, L. O., \& Bicudo, C. E. M. (2005). Structural and functional phytoplankton responses to nutrient impoverishment in mesocosms placed in a shallow eutrophic Reservoir (Garc, as Pond), São Paulo, Brazil. Hydrobiologia, 541, 71-85. DOI: 10.1590/S0100-

\section{9}

Crossetti, L. O., \& Bicudo, C. E. M. (2008). Adaptations in phytoplankton life strategies to imposed change in a shallow urban tropical eutrophic reservoir, Garças Reservoir, over 8 years. Hydrobiologia, 614(1), 91-105. DOI: 10.1007/s10750-008-9539-1

Cupertino, A., Gücker, B., Von Rückert, G., \& Figueredo, C. C. (2019). Phytoplankton assemblage composition as an environmental indicator in routine lentic monitoring: Taxonomic versus functional groups. Ecological Indicators, 101, 522-532. DOI: 10.1016/j.ecolind.2019. 01.054

Dos Santos, A., \& Calijuri, M. (1998). Survival strategies of some species of the phytoplankton community in the Barra Bonita Reservoir (Sao Paulo, Brazil). Hydrobiologia, 367(1-3), 139-152. DOI: 10.1023/a:1003276016149

Huichao-Dai, M. J., Jiang, D., \& Wang, L. (2013). Longitudinal hydrodynamic characteristics in reservoir tributary embayments and effects on algal blooms. PLoS ONE, 8(7), e68186. DOI: 10.1371/journal.pone.0068186

Edler, L. (ed). (1979). Recommendations on methods for marine biological studies in the Baltic Sea: phytoplankton and Chlorophyll. The Baltic Marine Biologist. University of Stockholm. Sweden.

Gomes Barbosa, L., Maia-Barbosa, P. M., \& Rodrigues-Barbosa, F. A. (2011). Vertical distribution of phytoplankton functional groups in a tropical shallow lake: driving forces on a diel sacel. Acta limnologica Brasiliensia, 23(1), 63-73. DOI: 10.4322 actalb.2011.020

Grill, G., Lehner, B., Thieme, M., Greenen, B., Antonelli, F., Tickner, D., \& Antonelli, F. (2019). Mapping the world's free-flowing rivers. Nature, 569(7755), 215-221. DOI: 10.1038/ s41586-019-1111-9

Grigorszky, I., Borics, G., Padisák, J., Totmeresz, B., Vasas, G., Nagy, S., \& Borbely, G. (2003). Factors controlling the occurrence of Dinophyta species in Hungary. Hydrobiologia, 506(1), 203-207. DOI: 10.1023/b:hydr. 0000008552.60232 .68

Guisande, C., Vaamonde, A., \& Barreiro, A. (2014). Paquete StatR - RWizard versión Beta 1.0. 
Hillebrand, H., Dürselen, C. D., Kirschtel, D. B., Pollingher, U., \& Zohary, T. (1999). Biovolume calculation for pelagic and benthic microalgae. Journal of Phycology, 35 (2), 403-424. DOI: 10.1046/j.1529-8817.1999.3520403.x

Hoyer, A. B., Moreno-Ostos, E., Vidal, J., Blanco, J. M., Palomino-Torres, R. L., Basanta, A., \& Escot, C. (2009). The influence of external perturbations on the functional composition of phytoplankton in a Mediterranean reservoir. Hydrobiologia, 636, 49-64. DOI: 10.1007/ s10750-009-9934-2

Hu, R., Han, B., \& Naselli-Flores, L. (2013). Comparing biological classifications of freshwater phytoplankton: a case study from South China. Hydrobiologia, 701(1), 219-233. DOI: 10.1007/s10750-012-1277-8

Jones, R. I. (2000). Mixotrophy in planktonic protists: an overview. Freshwater Biology, 45, 219226. DOI: 10.1046/j.1365-2427.2000.00672.x

Lira, G., Moura, A. N., Vilar, M. C. P., Cordeiro-Araujo, M. K., \& Bittencourt-Oliveira, M. C. (2014). Vertical and temporal variation in phytoplankton assemblages correlated with environmental conditions in the Mundaú reservoir, semi-arid northeastern Brazil. Brazilian Journal of Biology, 74(3), 93-102. DOI: 10.1590/1519-6984.27612

Litchman, E., Pinto, P. D., Edwards, K. F., Klausmeier, C. A., Kremer, C. T., \& Thomas, M. K. (2015). Global biogeochemical impacts of phytoplankton: a trait-based perspective. Journal of Ecology, 103, 1384-1396. DOI: $10.1111 / 1365-2745.12438$

López-Muñóz, M. T. (2015). Aspectos taxonómicos y ecológicos del fitoplancton eucariótico del embalse Río Grande II (Antioquia, Colombia). (Tesis de Doctorado. Universidad de Antioquia, Colombia).

Lund, J. W. G., Kipling, C., \& Le Cren, E. D. (1958). The inverted microscope method of estimating algal numbers and the statistical basis of estimations by counting. Hydrobiology, 11, 143-170. DOI: 10.1007/BF00007865

Mac Donagh, M. E., Casco, M. A., \& Claps, M. C. (2009). Plankton relationships under small water level fluctuations in a subtropical reservoir. Aquatic Ecology, 43, 371-381. DOI: 10.1007/s10452-008-9197-4
Magalhães, L., Machado-Rangel, L., De Melo Rocha, A., Cardoso, S. J., \& Sampiao Da Silva, L. H. (2021). Responses of morphology-based phytoplankton functional groups to spatial variation in two tropical reservoirs with long water residence time. Inland Waters, 11(1), 1-15. DOI: $10.1080 / 20442041.2020 .1745007$

Marques-Lopes, M. R., Bicudo, C. E., \& Ferragut, C. (2005). Short term and spatial and temporal variations of phytoplankton in a shallow tropical oligotrophic reservoir, southeast Brazil. Hydrobiologia, 542, 235-247. DOI: 10.1007/ s10750-004-833

Matsumura-Tundisi, T., Tundisi, J. G., Luzia, A. P., \& Degani, R. M. (2010). Occurrence of Ceratium furcoides (Levander) Langhans 1925 bloom at the Billings Reservoir, São Paulo State, Brazil. Brazilian Journal of Biology, 70(3), 825-829. DOI: 10.1590/S151969842010000400013

Modesto, M., Guillot, G., Donato, J. C., \& Ortegón, M. T. (2002). Dimensión fractal y relación área superficial/volumen de algas del fitoplancton de lagos colombianos. Caldasia, 24(1), 121-134. https://revistas.unal.edu.co/index.php/cal/ article/view/39437

Moreti, L. O. R., Martos, L., Bovoscomparin, V. M., \& Rodrigues, L. C. (2013). Spatial and temporal fluctuation of phytoplankton functional groups in a tropical reservoir. Acta Scientiarum. Biological Sciences, 35(3), 359366. DOI: 10.4025/actascibiolsci.v35i3.12998

Moura, A. N., Bittencourt-Oliveira, M. C., Dantas, E. W., \& Arruda-Neto, J. D. T. (2007). Phytoplanktonic associations: a tool to understand dominance events in a tropical Brazilian reservoir. Acta Botanica Brasilica, 21(3), 641-648. DOI: 10.1590/S0102-33062007000300011

Naselli-Flores, L. (2000). Phytoplankton assemblages in twenty-one Sicilian reservoirs: Relationships between species composition and environmental factors. Hydrobiologia, 424(1), 1-11. DOI: 10.1023/A:1003907124528

Naselli-Flores, L. \& Barone, R. (2003). Steadystate assemblages in a Mediterranean hypertrophic reservoir. The role of Microcystis ecomorphological variability in maintaining an apparent equilibrium. Hydrobiologia, 502, 133-143. DOI: 10.1023/B:HYDR. 
0000004276.11436 .40

Naselli-Flores, L. \& Barone, R. (2005). Water level fluctuations in Mediterranean reservoirs: setting a dewatering threshold as a management tool to improve water quality. Hydrobiologia, 548, 85-99. DOI: 10.1007/s10750-0051149-6

Naselli-Flores, L. (2014). Morphological analysis of phytoplankton as a tool to assess ecological state of aquatic ecosystems: The case of Lake Arancio, Sicily, Italy. Inland Waters, 4(1), 1526. DOI: $10.5268 / \mathrm{IW}-4.1 .686$

Naselli-Flores, L., \& Barone, R. (2000). Phytoplankton dynamics and structure: a comparative analysis in natural and manmade water bodies of different trophic state. Hydrobiologia, 438(1/3), 65-74. DOI: 10.1023/A: 1004109912119

Naselli-Flores, L., Termine, R., \& Barone, R. (2016). Phytoplankton colonization patterns. Is species richness depending on distance among freshwaters and on their connectivity?. Hydrobiologia, 764, 103-113. DOI: 10.1007/ s10750-015-2283-4

Ospina-Calle, L. M., \& Ramírez, J. J. (2017). Aspectos sucesionales de la comunidad fitoplanctónica en un embalse neotropical: embalse Riogrande II, Antioquia, Colombia. (Tesis de pregrado. Universidad de Antioquia, Colombia).

Padisák, J., Crossetti, L. O., \& Naselli-Flores, L. (2009). Use and misuse in the application of the phytoplankton functional classification: a critical review with updates. Hydrobiologia, 621(1), 1-19. DOI: 10.1007/s10750-008-9645-0

Palacio-Betancurt, H. M. (2014). Dinámica espacio-temporal de las cianobacterias en el embalse Riogrande II. (Tesis de doctorado. Universidad de Antioquia, Colombia).

Ramírez, J.J. (2000). Fitoplancton de agua dulce: aspectos ecológicos, taxonómicos y sanitarios. Editorial Universidad de Antioquia. Medellín. Colombia.

Rangel, L., Silva, L., Rosa, P., Roland, F., \& Huszar, Vera. (2012). Phytoplankton biomass is mainly controlled by hydrology and phosphorus concentrations in tropical hydroelectric reservoirs. Hydrobiologia, 693(1), 13-28, DOI: $10.1007 / \mathrm{s} 10750-012-1083-3$
Reynolds, C. S. (1993). Scales of disturbance and their role in plankton ecology. En: J. Padisak, C. S. Reynolds \& U. Sommer (eds.). Intermediate Disturbance Hypothesis in Phytoplankton Ecology. Developments in Hydrobiology 8 1. (pp. 157-171). Kluwer Academic Publishers, Dordrecht, Países Bajos.

Reynolds, C. S. (1988). Functional Morphology and adaptive strategies of freshwater phytoplankton. En: C. D. Sandgren (ed.). Growth and reproductive strategies of freshwater phytoplankton. (pp. 388-433). Cambridge University Press. Cambridge. Reino Unido.

Reynolds, C. S. (2006). Ecology of phytoplankton. Cambridge University Press. Cambridge. Reino Unido.

Reynolds, C. S., Huszar, V., Kruk, C., Naselli-Flores, L., \& Melo, S. (2002). Towards a functional classification of the freshwater phytoplankton. Journal of Plankton Research, 24(5), 417-428. DOI: 10.1093/plankt/24.5.417

Richerson, P. J., Armstrong, R., \& Goldman, C. R. (1970). Contemporaneous disequilibrium: a new hypothesis to explain the paradox of plankton. Proceedings of the National Academy of Sciences USA, 67(4), 1710-1714. DOI: 10.1073/pnas.67.4.1710

Rodrigues, L. C., Train, S., Pivato, B. M., Bovo-Scomparin, V. M., Borges, P. A. F., \& Jati, S. (2005). Assembléias fitoplanctônicas de trinta reservatórios do estado do Paraná. En: L. Rodrigues, S. M. Thomaz., A.A. Agostinho \& L. C. Gomes. (Ed.). Biocenoses em reservatórios: padrões espaciais e temporais. (pp. 57-72). Rima, São Carlos, Brasil.

Pivato, B. M., Train, S., \& Rodrigues, L. C. (2006). Dinâmica nictemeral das assembléias fitoplanctônicas em um reservatório tropical (reservatório de Corumbá, estado de Goiás, Brasil), em dois períodos do ciclo hidrológico. Biological Sciences, 28 (1), 19-29. DOI: 10.4025/actascibiolsci.v28i1.1054

Roldán, G., \& Ramírez, J. J. (2008). Fundamentos de limnología Neotropical. Editorial Universidad de Antioquia, Universidad Católica de Oriente y Academia Colombiana de Ciencias-ACCEFYN. Medellín. Colombia.

Ross, J. (1979). Prácticas de Ecología. Ediciones Omega S. A. Barcelona. España. 
Salmaso, N. (2003). Life strategies, dominance patterns and mechanisms promoting species coexistence in phytoplankton communities along complex environmental gradients. $\mathrm{Hy}$ drobiologia, 502(2), 13-36. DOI: 10.1023/B: HYDR.0000004267.64870.85

Salmaso, N., \& Tolotti, M. (2010). Other phytoflagellates and groups of lesser importance. En: G. E. Likens (ed.). Plankton of Inland Waters. (pp. 165-174). Amsterdam. Holanda.

Soares-Vieria, P. C., Lima-Cardoso, M. M., Alves, I. (2015). Vertical and temporal dynamics of phytoplankton associations and the applications of index assembly in tropical semi-arid autrophic resrevoir, northeastern Brazil. Acta Limnologica Brasiliensia, 27(1), 130-144. DOI: $10.1590 / \mathrm{S} 2179-975 X 2614$

Sokal, R., \& Rohlf, F. J. (1981). Biometry. Freeman. California. Estados Unidos.

Straskraba, M. \& Tundisi, J. G. (1999). Reservoir Water Quality Management: Guidelines of Lake Management. Vol. 9. Kusatsu: International Lake Environmental Committee.

Souza, D., Bueno, N. C., Bortolini, J. C., Rodrigues, L. C., Bovo-Scomparin, V. M., \& De Souza Franco, G. M. (2016). Phytoplankton functional groups in a subtropical Brazilian reservoir: responses to impoundment. Hydrobiologia, 779, 47-57. DOI: 10.1007/s10750-0162798-3

Train, S., Jati, S., Rodrigues, L. C., \& Pivato, B. M. (2005). Distribuição espacial e temporal do fitoplâncton em três reservatórios da bacia do rio Paraná. En: L. Rodrigues., S.M. Thomaz, A. A. Agostinho y L. C. Gomes (Ed.). Biocenoses em reservatórios: padrões espaciais e temporais. (pp. 12). Rima, São Carlos. Brasil. Thornton, K. W., Kimmel, B. L., \& Payne, F. E. (1990). Reservoir limnology: ecological perspectives. John Willey \& Sons. New Jersey. Estados Unidos.

Utermöhl, H. (1958). Zur Vervollkommung der quantitativen Phytoplankton Methodik. Internationale Vereinigung für Theoretische und Angewandte Limnologie: Verhandlungen, 9(1), 1-38. DOI: 10.1080/05384680.1958.11904091

Wang, L., Cai, Q., Xu, Y., Kong, L., Tan, L., Zhang, M. (2011). Weekly dynamics of phytoplankton functional groups under high water level fluctuations in a subtropical reservoir-bay. Aquatic Ecology, 45(2), 197-212. DOI: $10.1080 / 05384680.1958 .11904091$

Wetzel, R. G., \& Likens, G.E. (1991). Limnological analyses. Springer. Nueva York. Estados Unidos

Xiao, L. J., Wang, T., Hu, R., Han, B. P., Wang, S., Qian, X., \& Padisak, J. (2011). Succession of phytoplankton functional groups regulated by monsoonal hydrology in a large canyon-shaped reservoir. Water Research, 45(16), 5099-5109. DOI: 10.1016/j.watres.2011.07.012

Zarfl, C., Lumsdon, A. E., Berlekamp, J., Tydecks, L., \& Tockner, K. (2015). A global boom in hydropower dam construction. Aquatic Sciense, 77, 161-170. DOI: 10.1080/ 05384680.1958 .11904091

Zhang, H., Qi, W., John, R., Wang, W., Song, F., $\&$ Zhou, S. (2015). Using functional trait diversity to evaluate the contribution of multiple ecological processes to community assembly during succession. Ecography, 38, 1176-1186. DOI: $10.1111 /$ ecog.01123 